\title{
Investigating antimicrobial features and drug interactions of sedoanalgesics in intensive care unit: an experimental study
}

\author{
Ozge Unlu ${ }^{1}$, Emre Sertac Bingul ${ }^{2}$, Sevgi Kesici ${ }^{3 *}$, Mehmet Demirci ${ }^{4}$ \\ ${ }^{1}$ Istanbul Atlas University, Faculty of Medicine, Department of Medical Microbiology, Turkey \\ ${ }^{2}$ Recep Tayyip Erdogan University, Training and Research Hospital, Department of Anesthesiology and Reanimation, \\ Turkey \\ ${ }^{3}$ Health Sciences University, Hqamidiye Etfal Training and Research Hospital, Department of Anesthesiology and \\ Reanimation, Turkey \\ ${ }^{4}$ Kirklareli University, Faculty of Medicine, Department of Medical Microbiology, Turkey
}

*Sevgi Kesici: E-mail: md.kesici@mynet.com ; Tel.: +902124426649

Received: July 03, 2021; Revised: August 25, 2021; Available online: September 06, 2021

\begin{abstract}
Study Objective: Aim of this study was to evaluate antimicrobial effects and interaction between analgesic combinations of fentanyl citrate, dexmedetomidine hydrochloride and tramadol hydrochloride on Staphylococcus aureus, Klebsiella pneumoniae, Escherichia coli, Pseudomonas aeruginosa and Candida albicans which are some of the most common nosocomial infection related microorganisms. Design: In vitro prospective study. Setting: University Clinical Microbiology Laboratory. Measurements: In order to evaluate in vitro antimicrobial effects and interaction between analgesic combinations, tramadol hydrochloride, fentanyl citrate and dexmedetomidin were used against S. aureus ATCC 29213, K. pneumoniae, E. coli ATCC 25922, P. aeruginosa ATCC 27853 and C. albicans ATCC 10231 standard strains by microdilution method. Main Results: According to microdilution assays tramadol has shown the most efficient antimicrobial activity also it has been observed that $10 \mu \mathrm{g} / \mathrm{ml}$ concentrated dexmedetomidine has antimicrobial effects on S. aureus, $K$. pneumoniae and $P$. aeruginosa. Fentanyl has displayed evident inhibitory potency on the pathogens except for Klebsiella pneumoniae, nevertheless our predefined minimum concentration inhibited growth by $9.5 \%$. Fentanyl and dexmedetomidine together exhibited more antimicrobial effect on $P$. aeruginosa and $E$. coli growth. Additionally, when the three drugs examined together, microbial inhibition occurred more than expected on $E$. coli again and also on $C$. albicans growth. Conclusions: Our results revealed the antimicrobial properties and synergy with the different combinations of fentanyl, dexmedetomidine and tramadol against the most common nosocomial infection agents in the ICU. This is the first study in the literature looking into the microbial "interactions" of opioids and sedative drugs but more research is needed in order to define clinico-laboratory correlation.

(C)2021 by the authors. This article is an open-access article distributed under the terms and conditions of the Creative Commons Attribution license (http://creativecommons.org/licenses/by/4.0/).
\end{abstract}

\section{Keywords}

Sedoanalgesics; nosocomial agents; antimicrobial effects; drug interactions. 


\section{Introduction}

Intensive care patients often require sedation procedures for several reasons during their hospitalization. Since intensive care units (ICU) are prone to nosocomial infections; intravenous (IV) medications gain more importance in the aspect of microbial features. Contamination based infections may complicate clinical follow-up and little is known whether the sedative agents have antimicrobial properties. Among them, propofol is a well-studied drug that is now known to be inducing bacterial growth [1-4]. However, there are many other drug combinations used in routine practice to maintain sedation.

$\alpha-2$ selective blocker dexmedetomidine has become more popular and widely used for its less respiratory depressant effects instead of midazolam which triggers delirium in the elderly [5-7]. Many clinicians also add opioids to the treatment just to deepen sedation and fentanyl appears to be one of the most common choices due to its hemodynamic stable properties. There are studies comparing dexmedetomidine and midazolam from the microbiological aspect in the literature $[8,9]$. But since midazolam has gained a bad reputation for delirium triggering properties another sedative agent fentanyl is yet to be explored.

This study aimed to evaluate antimicrobial effects and interaction between analgesic combinations of fentanyl citrate, dexmedetomidine hydrochloride and tramadol hydrochloride on Staphylococcus aureus, Klebsiella pneumoniae, Escherichia coli, Pseudomonas aeruginosa and Candida albicans which are some of the most common nosocomial infection related microorganisms. These drugs were chosen for being the most frequently used infusion sedoanalgesics in our institute.

\section{Materials and methods}

\section{Tested sedoanalgesics}

Tramadol hydrochloride (ultramex), fentanyl citrate (talinat) and dexmedetomidin (hipnodex) were tested to reveal their antimicrobial activity and interaction between analgesic combinations.

\section{Bacterial strains}

S. aureus ATCC 29213, K. pneumoniae, E. coli ATCC 25922, P. aeruginosa ATCC 27853 and C. albicans ATCC 10231 standard strains, which are primary pathogens of bacteremia and sepsis were chosen in order to compare analgesics antimicrobial activity.

\section{Antibacterial broth test}

Antimicrobial activity of the selected analgesics was measured with microdilution method according to the instructions of Clinical Laboratory Standards Institute (CLSI). Tramadol hydrochloride (Abbott, Rocky Mount, NC, USA, supplied in a $2 \mathrm{ml}$ ampoule containing $50 \mu \mathrm{g} / \mathrm{ml}$ in sodium chloride solution) was diluted with Brain Heart Infusion Broth (BHI) to the final concentrations of $500 \mu \mathrm{g} / \mathrm{ml}, 250 \mu \mathrm{g} / \mathrm{ml}, 125 \mu \mathrm{g} / \mathrm{ml}, 62.5$ $\mu \mathrm{g} / \mathrm{ml}, 31.25 \mu \mathrm{g} / \mathrm{ml}$. Fentanyl citrate (Abbott, Rocky Mount, NC, USA, supplied in a $10 \mathrm{ml}$ ampoule containing $0,05 \mathrm{mg} / \mathrm{ml}$ in sodium chloride solution) was diluted with Brain Heart Infusion Broth (BHI) to the final concentrations of $5 \mu \mathrm{g} / \mathrm{ml}, 2.5 \mu \mathrm{g} / \mathrm{ml}, 1.25 \mu \mathrm{g} / \mathrm{ml}, 0.625 \mu \mathrm{g} / \mathrm{ml}, 0.3125 \mu \mathrm{g} / \mathrm{ml}$. Dexmedetomidine hydrochloride (Abbott, Rocky Mount, NC, USA, supplied in a $2 \mathrm{ml}$ ampoule containing $100 \mathrm{\mu g} / \mathrm{ml}$ in sodium chloride solution) was diluted with Brain Heart Infusion Broth (BHI) to the final concentrations of $10 \mu \mathrm{g} / \mathrm{ml}$, $5 \mu \mathrm{g} / \mathrm{ml}, 2.5 \mu \mathrm{g} / \mathrm{ml}, 1.25 \mu \mathrm{g} / \mathrm{ml}, 0.625 \mu \mathrm{g} / \mathrm{ml}$. Overnight broth cultures of S. aureus ATCC 29213, K. pneumoniae ATCC 13883, E. coli ATCC 25922, P. aeruginosa ATCC 27853 and C. albicans ATCC 10231 were adjusted to the turbidity of a $0.5 \mathrm{McF}$ arland standard. $10 \mu \mathrm{l}$ of each strain were inoculated to each well. Broths without any analgesic materials were served as controls for comparison. Plates incubated overnight and bacterial growth in each well were measured with Epoch spectrophotometer (Biotek, Germany) at $600 \mathrm{~nm}$. 
Interaction between analgesic combinations via broth microdilution method

Tramadol hydrochloride (TH), fentanyl citrate (FC) and dexmedetomidine hydrochloride (DH) combinations were diluted in Brain Heart Infusion Broth (BHI) with or without S. aureus ATCC 29213, $K$. pneumoniae ATCC 13883, E. coli ATCC 25922, P. aeruginosa ATCC 27853 and C. albicans ATCC 10231 were adjusted to the turbidity of a $0.5 \mathrm{McFarland}$ standard. $10 \mu \mathrm{l}$ of each strain were inoculated to each well. Broths without any analgesic materials were served as controls for comparison. Plates were incubated overnight and bacterial growth in each well were measured with Epoch spectrophotometer (Biotek, Germany) at $600 \mathrm{~nm}$. The preparation of the microdilution in microwells with single agent concentrations of the analgesic to determine the minimum inhibitory concentration (MIC), control wells and paired combinations of the analgesics were used in unique concentrations to determine the fractional inhibitory concentration (FIC). The value of the $\Sigma$ FIC index is then used to determine whether synergism, indifference or antagonism occurred between the antimicrobial agents and it was used to interpret the nature of the interactions: synergism $\leq 0.5$, indifference $>0.5$ to $\leq 4$, antagonism $>4$ according to American Society of Microbiology [10].

\section{Results}

\section{Antimicrobial activity for analgesics}

Antimicrobial susceptibility results revealed that $500 \mu \mathrm{g} / \mathrm{ml}$ tramadol hydrochloride inhibited the growth of $K$. pneumoniae $100 \%$. Also, inhibition rates for P. aeruginosa, S. aureus, E. coli, and C. albicans were 40, 35, 28.8 and $37.5 \%$, respectively. Moreover, the inhibitory effects of tramadol hydrochloride were observed at descending rates until the lowest concentration tested which was $31,25 \mu \mathrm{g} / \mathrm{ml}$ for $K$. pneumoniae, E. coli, and C. albicans.

In addition, $5 \mu \mathrm{g} / \mathrm{ml}$ fentanyl citrate inhibited the growth of S. aureus, P. aeruginosa, E. coli, and C. albicans at the following rates $38,13.3,4.5$ and $7.5 \%$, respectively. Also, fentanyl citrate did not show any inhibitory effect on the growth of $K$. pneumoniae at maximum concentration tested, whereas it inhibited growth $9.5 \%$ in its minimum concentration $(3,125 \mu \mathrm{g} / \mathrm{ml})$.

Moreover, $10 \mu \mathrm{g} / \mathrm{ml}$ dexmedetomidine had an inhibitory effect on the growth of $S$. aureus, $K$. pneumoniae and $P$. aeruginosa at the rates of 34,21 and $12.1 \%$, respectively. Also, dexmedetomidine did not show any inhibitory effect on the growth of E.coli at maximum concentration tested, whereas it inhibited growth $5 \%$ in its minimum concentration. Moreover, it has been observed that the growth of $C$. albicans inhibited $8.4 \%$ at the maximum concentration of the analgesic while it has been inhibited $28 \%$ at the minimum concentration tested.

All the results of microdilution assays for tramadol hydrochloride, fentanyl citrate and dexmedetomidine were given in Table 1, Table 2 and Table 3, respectively.

Table 1. Antimicrobial effects of tramadol hydrochloride in different concentrations against to tested microorganisms

\begin{tabular}{lccccc}
\hline Microorganisms & $\mathbf{5 0 0} \boldsymbol{\mu g} / \mathbf{m l}$ & $\mathbf{2 5 0} \boldsymbol{\mu g} / \mathbf{m l}$ & $\mathbf{1 2 5} \boldsymbol{\mu g} / \mathbf{m l}$ & $\mathbf{6 2 . 5} \boldsymbol{\mu g} / \mathbf{m l}$ & $\mathbf{3 1 . 2 5} \boldsymbol{\mu g} / \mathbf{m l}$ \\
\hline K. pneumoniae & $100.00 \%$ & $80.02 \%$ & $58.41 \%$ & $58.06 \%$ & $60.63 \%$ \\
P. aeruginosa & $40.03 \%$ & $0.00 \%$ & $0.00 \%$ & $0.00 \%$ & $0.00 \%$ \\
E. coli & $28.74 \%$ & $28.74 \%$ & $20.49 \%$ & $7.77 \%$ & $3.89 \%$ \\
S. aureus & $34.62 \%$ & $33.46 \%$ & $26.25 \%$ & $7.21 \%$ & $0.00 \%$ \\
C. albicans & $35.55 \%$ & $26.39 \%$ & $27.47 \%$ & $27.11 \%$ & $27.65 \%$ \\
\hline
\end{tabular}


Table 2. Antimicrobial effects of fentanyl citrate in different concentrations against to tested microorganisms

\begin{tabular}{lccccc}
\hline Microorganisms & $\mathbf{5 ~} \mathbf{~ g} / \mathbf{m l}$ & $\mathbf{2 . 5} \mathbf{\mu g} / \mathbf{m l}$ & $\mathbf{1 . 2 5} \boldsymbol{\mu g} / \mathbf{m l}$ & $\mathbf{0 . 6 2 5} \boldsymbol{\mu g} / \mathbf{m l}$ & $\mathbf{0 . 3 1 2 5} \boldsymbol{\mu g} / \mathbf{m l}$ \\
\hline K. pneumoniae & $0.00 \%$ & $4.09 \%$ & $6.78 \%$ & $6.89 \%$ & $9.58 \%$ \\
P. aeruginosa & $13.34 \%$ & $8.67 \%$ & $4.85 \%$ & $1.04 \%$ & $0.69 \%$ \\
E. coli & $4.59 \%$ & $6.60 \%$ & $6.95 \%$ & $6.48 \%$ & $7.77 \%$ \\
S. aureus & $38.22 \%$ & $39.64 \%$ & $34.75 \%$ & $21.24 \%$ & $1.93 \%$ \\
C. albicans & $7.54 \%$ & $26.57 \%$ & $36.45 \%$ & $38.24 \%$ & $30.70 \%$ \\
\hline
\end{tabular}

Table 3. Antimicrobial effects of dexmedetomidine in different concentrations against to tested microorganisms

\begin{tabular}{lccccc}
\hline Microorganisms & $\mathbf{1 0} \mathbf{~ g} / \mathbf{m l}$ & $\mathbf{5 ~} \mathbf{~ g} / \mathbf{m l}$ & $\mathbf{2 . 5} \boldsymbol{\mu g} / \mathbf{m l}$ & $\mathbf{1 . 2 5} \mathbf{~ g g} / \mathbf{m l}$ & $\mathbf{0 . 6 2 5} \boldsymbol{\mu g} / \mathbf{m l}$ \\
\hline K. pneumoniae & $21.14 \%$ & $8.53 \%$ & $3.04 \%$ & $0.00 \%$ & $0.00 \%$ \\
P. aeruginosa & $12.13 \%$ & $13.00 \%$ & $12.11 \%$ & $5.37 \%$ & $0.69 \%$ \\
E. coli & $0.00 \%$ & $6.83 \%$ & $6.12 \%$ & $3.42 \%$ & $4.83 \%$ \\
S. aureus & $33.98 \%$ & $26.00 \%$ & $33.20 \%$ & $20.21 \%$ & $6.31 \%$ \\
C. albicans & $8.44 \%$ & $28.73 \%$ & $33.75 \%$ & $24.78 \%$ & $20.83 \%$ \\
\hline
\end{tabular}

According to microdilution results, tramadol hydrochloride had the most powerful effect against bacterial growth. Moreover, antimicrobial susceptibility results suggest that antimicrobial effect of analgesics vary according to the type of the analgesic, pathogen and the concentration of the material. Also, higher concentration does not always result higher antimicrobial effect.

\section{Interaction between analgesic combinations}

SFIC index for tramadol hydrochloride, fentanyl citrate and dexmedetomidine hydrochloride combinations against standard strains were shown in Table 4.

Table 4. ¿FIC index for tramadol hydrochloride, fentanyl citrate and dexmedetomidine hydrochloride combinations against standard strains.

\begin{tabular}{ccccc}
\hline$\sum$ FIC & TH+FC & TH+DH & FC + DH & TH+FC+DH \\
\hline C. albicans & 1.17 & 0.58 & 1.53 & 0.45 \\
E. coli & 1.46 & 0.64 & 0.23 & 0.29 \\
S. aureus & 1.17 & 0.90 & 1.34 & 0.89 \\
K. pneumoniae & 0.72 & 0.90 & 3.23 & 0.53 \\
$\boldsymbol{P}$. aeruginosa & 1.69 & 1.60 & 0.43 & 0.74 \\
\hline
\end{tabular}

* Tramadol hydrochloride (TH), fentanyl citrate (FC) and dexmedetomidine hydrochloride (DH)

When the interactions between analgesic materials were analyzed, it was found that $\mathrm{FC}+\mathrm{DH}$ combination had a synergistic antimicrobial effect on the growth of $E$. coli and $P$. aeruginosa standard strains. Moreover, it has been observed that $\mathrm{TH}+\mathrm{FC}+\mathrm{DH}$ combination had a synergistic antimicrobial effect on E. coli and C. albicans standard strains.

\section{Discussion}

In this study, we have observed that $10 \mu \mathrm{g} / \mathrm{ml}$ concentrated dexmedetomidine has antimicrobial effects on S.aureus, $K$. pneumoniae and $P$. aeruginosa. These findings were slightly different from a previous study in which researchers have established the same activity with higher $(32 \mu \mathrm{g} / \mathrm{ml})$ concentrations [10]. Even though dexmedetomidine is a more preferred sedative drug than midazolam recently; antimicrobial effects were not described as well as it has been studied for midazolam. Dexmedetomidine seems to inhibit bacterial growth when prepared in correct concentrations. Commonly used infusions are prepared at a concentration of $4 \mu \mathrm{g} / \mathrm{ml}$ and according to our results, $10 \mu \mathrm{g} / \mathrm{ml}$ infusion fluids might be sufficient enough 
to prevent bacterial colonization.

One should declare that Keles et al. [9] have not found any antimicrobial effect of dexmedetomidine. However, this difference may be caused by the chosen microbiological assays. We have performed spectrophotometry after microdilution in $\mathrm{BHI}$ which is more sensitive than incubation in blood agar that Keles and her colleagues chose.

Dexmedetomidine hydrochloride does not contain preservatives or stabilizers. Therefore the inhibitor effect might be related to its chemical structure. The actual mechanism is unclear and also no microbial growth due to dexmedetomidine was declared in the literature until now [8,9]. In addition to its antimicrobial effect, Taniguchi et al. [11] have demonstrated anti-inflammatory features of this drug in rats. According to this study, $\alpha 2$-adrenoceptor agonist has modulated interleukin and TNF- $\alpha$ responses reducing excessive neutrophil accumulation on lung tissue. With that knowledge, dexmedetomidine might be a more appropriate drug than midazolam in septic patients who are in need of analgesia and sedation which requires further clinical studies.

Fentanyl citrate has displayed evident inhibitory potency on the pathogens except for Klebsiella pneumoniae, nevertheless our predefined minimum concentration inhibited growth by $9.5 \%$. To our knowledge, there are few studies focused on fentanyl's antimicrobial properties and some of them oppose our results [12]. For instance; Rota et al. [13] were not able to demonstrate the inhibiting effect of fentanyl. In contrast, Graystone et al. [14] have investigated 15 medications that are used in ICU and concluded all of them except propofol were bactericidal including fentanyl. Based on spectrophotometric calculations our study method was not designed to investigate the bactericidal effects of the drugs. However, as interpreted in the mentioned studies, we may propose specific assumptions. Fentanyl citrate ampoules contain 500 $\mu \mathrm{g} / 10 \mathrm{ml}$ which is a large dose for one use only. Therefore, multi-dosing with the same syringe is a quite common application practice in the ICU settings [12-15]. It could be argued that the drug would degrade and become susceptible to contamination during the time kept at room temperature in the same syringe. However, fentanyl is a physicochemically stable drug and can be stored in the syringe up to 1 week as a mixture with other drugs such as midazolam under $32{ }^{\circ} \mathrm{C}[16]$. Refilling of the syringes with this drug does not cause microbial contamination as observed in one prospective longitudinal study [12]. The reliability of multi-dosing with the same syringe is often questioned by the clinicians and considering our results this approach seems adequate. Perhaps further clinical studies are required.

A fentanyl analog "remifentanil" was investigated by Apan et al. [17] in the same manner. They have observed similar microbial inhibition on S. aureus, $P$. aeruginosa, $E$. coli and $C$. albicans depending on the concentration. However, this effect was attributed to "glycine" which is present in remifentanil ampoules as a preservative. It might be possible that glycine has bacteriostatic potency but since both remifentanil and fentanyl are 4-anilidopiperidine structured drugs, further evaluations are needed including also alfentanil and sufentanil $[18,19]$. Fentanyl citrate does not contain preservatives.

Tramadol is known to be a "good" analgesic that has few respiratory and hemodynamic adverse effects [20]. Despite being a weak opioid analgesic, it provides adequate analgesia especially in elder patients who are prone to cognitive dysfunction [21]. Therefore, it is mostly used for the postoperative period especially after orthopedic surgery in intensive care units. Many clinicians do not reckon tramadol as a sedative agent. But when combined with dexmedetomidine, these drugs show remarkable synergistic effects [20]. As a striking result of our study tramadol has shown the most efficient antimicrobial activity. From this aspect preparing combined infusions of dexmedetomidine and tramadol is rather considerable due to their fewer side-effect properties. It is worth noting that the synergy occurs only on sedation and analgesia but not on 
bacteriostatic activity. To the best of our knowledge, this is the only study examining antimicrobial features of tramadol.

Intravenous treatments are generally applied via central venous catheters in the ICUs. Occasionally multiple sedative drugs are needed to be given from the same catheter line and usually infused at lower rates. Whether these drug combinations covering the same line attenuate catheter related nosocomial infections is questionable. We have observed evident synergy with the different combinations against particular microorganisms in our study. Fentanyl and dexmedetomidine together exhibited more antimicrobial effects on $P$. aeruginosa and $E$. coli growth. Additionally, when the three drugs were examined together, microbial inhibition occurred more than expected on $E$. coli again and also on $C$. albicans growth. This interaction needs to be evaluated more. Since this is an in vitro study, in vivo examinations are necessary to adapt clinical practice. As far as we know there is no other study in the literature looking into the microbial "interactions" of opioids and sedative drugs. We believe these interactions are remarkably important which may affect routine IV therapy and shape the practitioner's approach. As a well-known fact, critical patients receiving total parenteral nutrition via the central venous catheters are at great risk for candidemia. The combination of TH, FC and DH infusion is worth investigating from this aspect. It is difficult to come to a conclusion with our laboratory results for sure but further in vitro and in vivo studies may reveal clinically significant interactions.

Perhaps the most important limitation of our study was the lack of hourly made spectrophotometric calculations. Therefore we could not demonstrate microbial inhibition or growth trend during 24 hours. Interestingly, Graystone et al. [14] have observed an increase in S. aureus number during the first 4 hours which declined later with all opioids except fentanyl. Pyrogenic reactions are reported because of extrinsically contaminated fluids even if there is no bacteremia [22]. Bacterial load in infusion fluids may be reduced by choosing the appropriate agent and concentration to enhance patient comfort in both sedation and anti-inflammatory meanings.

\section{Conclusions}

We have observed definite antimicrobial properties and synergy with the different combinations of fentanyl, dexmedetomidine and tramadol against the most common nosocomial infection agents in the ICU. This is the first study in the literature looking into the microbial "interactions" of opioids and sedative drugs but more research is needed in order to define clinico-laboratory correlation.

Limitations of this work are: (i) the study is in vitro study and (ii) clinical practice is required.

Conflict of interest: The authors declare no conflict of interest.

\section{References}

[1] M.B. Sosis, B. Braverman. Growth of Staphylococcus aureus in four intravenous anesthetics. Anesth. Analg. 77 (1993) 766-768.

[2] C.B. Berry, T. Gillespie, J. Hood, N.B. Scott. Growth of micro-organisms in solutions of intravenous anaesthetic agents. Anaesthesia 48 (1993) 30-32. https://doi.org/10.1111/j.1365-2044.1993.tb06786.x

[3] J. Crowther, J. Hrazdil, D.T. Jolly, J.C. Galbraith, M. Greacen, M. Grace. Growth of microorganisms in propofol, thiopental, and a 1:1 mixture of propofol and thiopental. Anesth. Analg. 82 (1996) 475-478. https://dx.doi.org/10.1097/00000539-199603000-00008. 
[4] D.V. Thomas. Propofol supports bacterial growth. Br. J. Anaesth. 66 (1991) 274. https://dx.doi.org/ 10.1093/bja/66.2.274-a.

[5] Schroeder ME. Delirium in the Elderly. In: Pain [Internet]. Cham: Springer International Publishing; 2019 [cited 2019 Jul 10]. p. 1117-20. Available from: http://link.springer.com/10.1007/978-3-31999124-5 238.

[6] F. Santana Santos, L.O. Wahlund, F. Varli, I. Tadeu Velasco, M. Eriksdotter Jonhagen. Incidence, clinical features and subtypes of delirium in elderly patients treated for hip fractures. Dement. Geriatr. Cogn. Disord. 20 (2005) 231-237. https://dx.doi.org/ 10.1159/000087311.

[7] N. Bhana, K.L. Goa, K.J. McClellan. Dexmedetomidine. Drugs 59 (2000) 263-268; discussion 269-270. https://dx.doi.org/ 10.2165/00003495-200059020-00012.

[8] H. Ayoglu, C. Kulah, I. Turan. Antimicrobial effects of two anaesthetic agents: dexmedetomidine and midazolam. Anaesth. Intensive Care 36 (2008) 681-684. https://dx.doi.org/ 10.1177/0310057X08036$\underline{00508 .}$.

[9] G.T. Keleş, S. Kurutepe, D. Tok, H. Gazi, G. Dinç. Comparison of antimicrobial effects of dexmedetomidine and etomidate-lipuro with those of propofol and midazolam. Eur. J. Anaesthesiol. 23 (2006) 1037-1040. https://dx.doi.org/10.1017/S0265021506000949.

[10] M.G. Botelho. Fractional inhibitory concentration index of combinations of antibacterial agents against cariogenic organisms. J. Dent. 28 (2000) 565-570. https://dx.doi.org/10.1016/s0300-5712(00)00039-7.

[11] T. Taniguchi, Y. Kidani, H. Kanakura, Y. Takemoto, K. Yamamoto. Effects of dexmedetomidine on mortality rate and inflammatory responses to endotoxin-induced shock in rats. Crit. Care Med. [Internet] 32 (2000) 1322-1326. https://doi.org/10.1097/01.ccm.0000128579.84228.2a.

[12] G. Morgan. Microbial growth in a mixture of hyperbaric bupivacaine and fentanyl prepared in a multi-dose syringe in the operating theatre environment. South African J. Anaesth. Analg. 16 (2010) 17-21. https://doi.org/10.1080/22201173.2010.10872661.

[13] S. Rota, K. Kaya, O. Timliothlu, O. Karaca, S. Yzdep, E. Ocal. Do the opioids have an antibacterial effect. Can. J. Anaesth. 44 (1997) 679-680. https://dx.doi.org/ 10.1007/BF03015460.

[14] S. Graystone, M.F. Wells, D.J. Farrell. Do intensive care drug infusions support microbial growth. Anaesth. Intensive Care 25 (1997) 640-642. https://dx.doi.org/ 10.1177/0310057X9702500608.

[15] S. Segal, A. Gunawan, D.H. McLaughlin, E. Palavecino. Microbial stability of syringes of anesthetic drugs prepared in the operating room. J. Clin. Anesth. 55 (2019) 20-23. https://dx.doi.org/ 10.1016/i.jclinane.2018.12.029.

[16] G.M. Peterson, K.A. Miller, J.G. Galloway, P.F. Dunne. Compatibility and stability of fentanyl admixtures in polypropylene syringes. J. Clin. Pharm. Ther. 23 (1998) 67-72. https://dx.doi.org/ 10.1046/i.1365-2710.1998.00141.x.

[17] T.Z. Apan, A. Apan, S. Sahin, M. Cakirca. Antibacterial activity of remifentanil and mixtures of remifentanil and propofol. J. Clin. Anesth. 19 (2007) 346-350. https://dx.doi.org/10.1016/j.jclinane.2007.02.005.

[18] A. Obayashi, S. Oie, A. Kamiya. Microbial viability in preparations packaged for single use. Biol. Pharm. Bull. 26 (2003) 667-670. https://dx.doi.org/10.1248/bpb.26.667.

[19] R.S. Vardanyan, V.J. Hruby. Fentanyl-related compounds and derivatives: current status and future prospects for pharmaceutical applications. Future Med. Chem. 6 (2014) 385-412. https://dx.doi.org/10.4155/fmc.13.215.

[20] C.R. Lee, D. McTavish, E.M. Sorkin. Tramadol. A preliminary review of its pharmacodynamic and pharmacokinetic properties, and therapeutic potential in acute and chronic pain states. Drugs 46 (1993) 313-340. https://dx.doi.org/10.2165/00003495-199346020-00008.

[21] E. Freye, J.V. Levy. The effects of tramadol on pain relief, fast EEG-power spectrum and cognitive function in elderly patients with chronic osteoarthritis (OA). Acute Pain 8 (2006) 55-61. https://doi.org/10.1016/i.acpain.2006.03.001. 
[22] S.N. Bennett, M.M. McNeil, L.A. Bland, M.J. Arduino, M.E. Villarino, D.M. Perrotta, D.R. Burwen, S.F. Welbel, D.A. Pegues, L. Stroud. Postoperative infections traced to contamination of an intravenous anesthetic, propofol. N. Engl. J. Med. 333 (1995) 147-154. https://dx.doi.org/10.1056/NEJM199507203330303.

C 2021 by the authors; licensee IAPC, Zagreb, Croatia. This article is an open-access article distributed under the terms and conditions of the Creative Commons Attribution license (http://creativecommons.org/licenses/by/3.0/) (cc) EY $_{\mathbf{E Y}}$ 